\title{
Le contrôle des différences en Europe : l'inclusion et l'exclusion comme logiques sécuritaires et économiques
}

Mike King

\section{(2) OpenEdition}

Journals

Édition électronique

URL : http://journals.openedition.org/conflits/361

DOI : $10.4000 /$ conflits.361

ISSN : $1777-5345$

Éditeur :

CCLS - Centre d'études sur les conflits lilberté et sécurité, L'Harmattan

Édition imprimée

Date de publication : 15 octobre 1997

ISSN : 1157-996X

\section{Référence électronique}

Mike King, «Le contrôle des différences en Europe : l'inclusion et l'exclusion comme logiques sécuritaires et économiques », Cultures \& Conflits [En ligne], 26-27 | automne 1997, mis en ligne le 15 mars 2006, consulté le 30 mars 2021. URL : http://journals.openedition.org/conflits/361 ; DOI : https://doi.org/10.4000/conflits.361

Ce document a été généré automatiquement le 30 mars 2021.

Creative Commons License 


\title{
Le contrôle des différences en Europe : l'inclusion et l'exclusion comme logiques sécuritaires et économiques
}

\author{
Mike King
}

\section{Mike KING ${ }^{1}$}

2 Vers une critique du débat sur l'" Europe Forteresse " Le débat actuel sur l'évolution des frontières européennes s'articule, d'une part, autour d'une tendance à l'ouverture des frontières extérieures et, d'autre part, autour de l'effet direct de celle-ci, à savoir l'introduction de " mesures de contrôle compensatoires "2. Au niveau de la recherche sur les immigrés, les réfugiés et les demandeurs d'asile, ce débat est centré sur un concept que l'on désigne d'habitude par le terme d'" Europe Forteresse ". Une des difficultés que pose cette notion est la généralisation de la référence à un espace unique, l'espace Schengen et l'Union européenne, au sein d'une Europe globale. Une autre vient de la focalisation du débat sur les contrôles d'exclusion, les politiques et leurs effets extérieurs à cet espace. De ce fait, l'Europe Forteresse a été décrite surtout comme une structure de refoulement des immigrants, des réfugiés et des demandeurs d'asile; une citadelle dont le pont-levis est fermé, un espace géopolitique aux portes obstinément closes, un rideau de fer. La présente étude soutient l'idée d'une réalité plus complexe dans la mesure où nous remettons en cause ce concept per se d'Europe Forteresse, et notamment la nature de la trame d'exclusion de cette forteresse ${ }^{3}$. Une approche plus nuancée de l'Europe Forteresse nécessiterait peut-être de la reconceptualiser à partir des processus dynamiques d'inclusion et d'exclusion. Si nous devions catégoriser le champ de l'inclusion, nous pourrions nous référer à l'incorporation, la convergence et l'harmonisation générale des organismes intergouvernementaux, des forums, des alliances et des associations. À l'inverse, catégoriser les processus d'exclusion pourrait se faire à travers les contrôles de routine et la notion de cordon sanitaire. Une telle approche ne ferait guère avancer le débat. Car, elle 
substitue à une Europe Forteresse de l'exclusion une trame d'exclusion et d'inclusion dont la ligne de partage est très nette. Nous soutenons, au contraire, que nous sommes confrontés à des séries de contrôles d'inclusion et d'exclusion simultanés. Ce qui se passe actuellement en Europe (et pas uniquement au sein de l'espace Schengen ou d'une Europe Forteresse) correspond à des processus bien plus complexes, qui doivent sans doute être reliés à des logiques sécuritaires et économiques, probablement plus complémentaires que contradictoires. En ce sens, ces processus à l'oeuvre impliquent des séries d'inclusions et d'exclusions différentielles parmi les groupes visés: les réfugiés, les demandeurs d'asile, les immigrants ${ }^{4}$. Les zones de transit et l'effet " domino " Nous avons déjà suggéré ailleurs 5 que le développement de " zones tampons " pour les réfugiés et les demandeurs d'asile correspond à l'inclusion de différentes formes de travail ${ }^{6}$. Les mesures d'exclusion, auxquelles nous faisons référence dans ce contexte, comprennent la désignation de pays d'origine ou de transit dits " sûrs ", dans lesquels, d'après les États membres ou les pays d'un groupe géopolitique, il n'existe pas, en général, de risques sérieux de persécution. Figurent parmi ceux-ci, les pays signataires de la Convention de Genève sur les réfugiés de 1951 et de la Convention européenne des Droits de l'Homme. En effet, sur cette base, l'article 16a de la Constitution allemande, qui a pris effet le 1er juillet 1993, stipule que les personnes faisant l'objet de persécutions politiques qui entrent " à partir d'un pays de l'Union européenne, ou d'un État tiers, garantissant l'application du traité concernant le statut des réfugiés et de la Convention de sauvegarde des Droits de l'Homme et des Libertés Fondamentales, ne bénéficient pas du droit d'asile ${ }^{7}$ ". Ceci signifie que l'Allemagne est protégée, au moins en théorie, par un " bouclier " externe, constitué par les pays sûrs, les États de l'Union européenne, de l'EFTA et les pays du groupe de Visegrád8. Cette notion de " pays de transit sûr ", auquel le réfugié adresse sa demande d'asile selon le principe du " premier point d'entrée ", repose dans la Convention de Dublin (1990) sur la règle de la demande unique, qui bloque toute demande à un autre pays membre. Suivant ces dispositions, tout demandeur d'asile, originaire d'un pays sûr ou en transit dans un tel pays, qui dépose sa demande à un pays membre de l'UE, est tenu de réfuter la présomption de sûreté, sinon sa demande sera rejetée comme étant manifestement infondée ${ }^{9}$. Les pratiques des pays sûrs ont fait l'objet des critiques de la Commission des Réfugiés des Nations Unies, comme étant contraires à l'esprit de la Convention de Genève $^{10}$. Leur application semble d'ailleurs susciter de plus en plus de problèmes ${ }^{11}$. À cette réglementation restrictive, relative aux pays sûrs, à la demande unique et aux demandes rejetées comme infondées (intégrées dans l'Asylum and Immigration Act 1993 du Royaume-Uni) s'ajoutent les contrôles d'exclusion, comportant des sanctions pour les convoyeurs et des accords de réadmission. * Concernant les " sanctions aux transporteurs ", l'article 26 de la convention d'application de l'accord de Schengen (1990) prévoit : Les parties contractantes s'engagent à introduire dans leur législation nationale les règles suivantes: $1(b)$ Le transporteur est tenu de prendre toutes les mesures nécessaires pour s'assurer que l'étranger transporté par voie aérienne ou maritime est en possession des documents de voyage requis pour l'entrée sur les territoires des Parties contractantes. 2) [...].instaurer des sanctions à l'encontre des transporteurs qui acheminent par voie aérienne ou maritime d'un État tiers vers leur territoire, des étrangers qui ne sont pas en possession des documents de voyage requis $^{12}$. 3) Les dispositions du paragraphe $1(\mathrm{~b})$ et du paragraphe 2 s'appliquent aux transporteurs de groupes assurant des liaisons routières internationales par autocar, à l'exception du trafic frontalier. L'adoption de telles mesures n'est pourtant pas propre 
aux pays signataires des accords de Schengen. La Grande-Bretagne, par exemple, a prévu des dispositions similaires, suite à l'adoption de l'Immigration (Carriers Liability) Act de $1987^{13}$ et de l'Asylum and Immigration Appeals Act de 1993 (section 12). Celles-ci s'appliquent aussi aux étrangers en transit. L'article 14 de la Convention de contrôle des personnes aux frontières extérieures de l'UE (1993) contient les mêmes dispositions que les accords de Schengen. Les sanctions applicables aux convoyeurs ont pour effet de poser une barrière d'exclusion aux réfugiés dépourvus de documents de voyage valides et de visas pour entrer par un pays tiers dans les pays signataires. Encore une fois, ces mesures semblent contrevenir aux dispositions de la Convention de Genève ${ }^{14}$. *Les accords de réadmission, quant à eux, consistent essentiellement en des accords bilatéraux, selon lesquels l'une des parties contractantes doit accepter les immigrants clandestins refoulés par l'autre. Ces clandestins sont des nationaux ou des personnes en transit sur le territoire de celle-ci. Ces accords présentent, néanmoins, des formes multiples : bilatéraux ou multilatéraux, unilatéraux ou réciproques, portant sur la réadmission des seuls nationaux ou de l'ensemble des demandeurs d'asile (rejetés ou autres) en transit et/ou des immigrants clandestins, et même sur la réadmission à des fins de transit. En même temps, ils peuvent être isolés ou être en " cascade ". Ils sont habituellement accompagnés soit d'aides soit de " compensations " matérielles envoyées par l'État qui refoule à l'État qui accueille. L'Allemagne, qui a établi ou négocié bilatéralement de tels accords avec les pays limitrophes à l'Est, constitue un cas typique en la matière ${ }^{15}$. Cependant, ces accords ne sont pas un phénomène nouveau en soi. La France, par exemple, a passé des accords bilatéraux avec l'Allemagne, l'Autriche, la Belgique, le Luxembourg et la Suisse dès les années 1960, prévoyant " la réadmission des personnes expulsées ou refoulées ", qu'elles soient des nationaux ou des étrangers ${ }^{16}$. Parmi les éléments nouveaux des années 1990, nous pouvons citer les accords de la deuxième génération, qui comportent : a) l'extension des refoulements, lesquels, visant initialement le retour des étrangers " entrés illégalement ", concernent à présent tout résident en situation irrégulière ${ }^{17}$; b) l'établissement d'accords similaires avec les pays d'Europe centrale et de l'Est. En outre, ces accords sont désormais à sens unique : des pays de l'Europe de l'Ouest vers ceux d'Europe centrale et de l'Est. En effet, Spencer affirme que l'accord multilatéral entre les États de Schengen et la Pologne, en 1991, a été le précurseur de nombreux accords entre les États de l'UE et les États intermédiaires, situés entre eux et les pays producteurs de réfugiés ${ }^{18}$. De plus, ces accords étant maintenant renforcés par des aides financières, il semble que certains soient établis simplement dans le but de faire transiter les personnes refoulées sur le territoire des pays concernés ${ }^{19}$. Vraisemblablement, les prochains accords d'association entre l'UE et des pays tiers, portant sur des questions liées à l'emploi (voir plus loin), seront conditionnés par l'intégration d'accords de réadmission ${ }^{20}$. En 1995, plus de 120 accords de réadmission ont été négociés ou signés. Ils se partagent équitablement entre pays hors de l'UE et entre ceux-ci et les membres de l'UE ${ }^{21}$. La question reste entière de savoir si ces accords sont de pure forme ou effectivement mis en pratique. Bien sûr, il semble au moins que certains pays d'Europe de l'Ouest les appliquent avec des pays d'Europe centrale et de l'Est. Ainsi, sur cette base ${ }^{22}$, entre 1993 et 1995 approximativement 85000 personnes ont été refoulées par avion vers la Roumanie. En vertu de l'accord germano-polonais de mai 1993, la Pologne a accepté le retour de 10000 de ses nationaux, puis leur retour en nombre illimité ${ }^{3}$. L'effet de ces accords de réadmission et de la désignation de pays d'origine et de transit sûrs, en particulier du groupe de Visegrád ${ }^{24}$ est que ces États agissent de facto comme une zone 
tampon entre l'Europe de l'Ouest et de l'Est, pour préserver l'Occident de la " menace de l'Est ", liée aux réfugiés et aux demandeurs d'asile. Ces contrôles d'exclusion, appliqués à l'Europe centrale, provoquent une " poussée vers l'Est ". En d'autres termes, la frontière extérieure de la zone tampon s'étend ou se déplace vers l'Est, souvent à la limite du groupe de Visegrád, et déborde déjà en Bulgarie et en Roumanie. La Pologne et la Bulgarie ont signé un accord de réadmission en cascade en août 1993, qui stipule que la Bulgarie accepte le retour de " tous les demandeurs d'asile bulgares qui sont refoulés par l'Allemagne et renvoyés en Pologne, en vertu de l'accord de réadmission germano-polonais de mai 1993, et de tous les Bulgares interpellés pour tentative d'entrée illégale en Pologne "25. De même, en août 1993, la République Tchèque et la Pologne ont signé un accord similaire, qui concernait notamment le retour, via la Pologne, d'immigrants clandestins roumains entrés en République Tchèque $^{26}$. Ensuite, on a essayé de lier ces accords à des aides financières afin de renforcer la frontière extérieure qui est en modification constante. Par exemple, malgré une relative " invisibilité " 27 de la ligne frontalière entre l'Europe centrale et occidentale, l'accord bilatéral germano-polonais prévoyait l'octroi " d'une aide financière pour améliorer le contrôle à la frontière avec la Biélorussie et l'Ukraine ${ }^{28}$ ". L'effet " domino "29 des accords de réadmission a amené les pays de transit en Europe centrale à négocier des accords de réadmission symétriques avec leurs voisins de l'Est ${ }^{30}$. Une Europe Forteresse? Effet pervers et harmonisation En plus de l'effet " domino " des contrôles d'exclusion, " l'intensification " des exclusions, devenue bien réelle dans certains États européens, semble, du moins dans un premier temps, créer un effet pervers plutôt qu'apporter une solution au problème ${ }^{31}$. Avant de le démontrer, nous devons néanmoins souligner brièvement certaines réalités de la situation des demandes d'asile, en nous référant de nouveau à l'Allemagne. D'abord, ces demandes ont connu une forte hausse annuelle entre 1977 et 1991, en passant de 16410 à 256 110. Comparé au nombre des demandes déposées en 1991 dans d'autres pays, 70000 aux États-Unis, 57000 en Grande-Bretagne et 5000 au Danemark, le cas de l'Allemagne paraît totalement disproportionné. La même situation peut être constatée en comparant le rapport entre le nombre des demandes et la population globale. Cette même année, il y eut en Allemagne ${ }^{32}$ demandes pour 10000 habitants, contre 3 aux États-Unis, 10 en Grande-Bretagne et 9 au Danemark ${ }^{33}$. Le pourcentage variable de réponses favorables réduirait certainement ces résultats. En Allemagne, seulement 3\% des demandes traitées en 1993 ont abouti à une reconnaissance du statut de réfugié dès le premier examen33. En Grande-Bretagne, le taux est inférieur à $3 \%$, même si $44 \%$ ont été acceptées au titre de " l'autorisation exceptionnelle de séjour " ${ }^{34}$. Le nombre de demandes d'asile en Allemagne en 1992 était de 437996 (représentant 79\% du total de l'UE). Par comparaison, la Grande-Bretagne ne comptait seulement que 24600 demandes. La majorité des demandes d'asile faites à la CEE (396 720) provenait de pays européens. Le second continent d'origine était l'Afrique, avec 103758 demandes, et le troisième l'Asie, avec 164148 demandes $^{35}$. Les statistiques pour le premier semestre de 1996 montrent que ce nombre est constant en Grande-Bretagne (14 860), tandis qu'en Allemagne il connaît une baisse significative (57 104), représentant 51\% de l'ensemble des demandes déposées dans l'UE ${ }^{36}$. L'entrée en vigueur de la loi sur l'asile en Autriche, en juin 1992, a produit une réduction du nombre de demandeurs d'asile de presque $80 \%$ pendant la première moitié de 1993. De même, en Allemagne, les six premiers mois de restriction de l'admissibilité des demandes d'asile, en vertu du nouvel amendement (A16a) de la Constitution (1er juillet 1993), ont été marqués par une baisse de moitié du 
nombre des demandes ${ }^{37}$. Les paramètres économiques et politiques déterminants de la variation des demandes d'asile n'ont vraisemblablement pas diminué pendant la même période, ce qui nous conduit à poser, en comparaison avec le problème de l'augmentation des contrôles dans les stades anglais, qui ont entraîné le déplacement $\mathrm{du}$ " hooliganisme " vers d'autres lieux, la question du déplacement des demandeurs d'asile vers d'autres pays. En août 1993, par exemple, le ministère de la Justice néerlandais a attribué l'augmentation du nombre des demandeurs d'asile aux " attaques contre les étrangers " en Allemagne mais aussi à la nouvelle loi sur l'Asile ${ }^{38}$. Cet effet de " déplacement " risque de devenir la principale justification des restrictions futures, comme l'indique la suggestion de l'ancien ministre français de l'Intérieur, C. Pasqua, selon lequel l'amendement A16a allemand " pourrait conduire à une augmentation considérable des demandes d'asile en France "39. Il est difficile de connaître la réalité de ces " déviations ", mais on peut s'attendre à " une harmonisation " du renforcement des contrôles d'exclusion en Europe, lesquels ne découlent pas simplement de l'exemple allemand ${ }^{39}$. Bien sûr, ces contrôles ne sont pas exercés envers les seuls réfugiés et demandeurs d'asile. Les pays signataires de la Convention d'application des accords de Schengen ont dressé une liste de 126 pays soumis au visa obligatoire, puis ont proposé une seconde de 129 pays $^{40}$. L'harmonisation future est inscrite dans la nouvelle Convention sur le contrôle des personnes aux frontières extérieures (1993). L'article 17 prévoit que " les États membres entreprennent l'harmonisation progressive de leurs politiques de visa ". Les ministres responsables de l'UE l'ont noté, lors de la réunion de juin 1993, qu'il y avait 92 pays tiers dont les ressortissants étaient obligés de détenir un visa, conformément à la législation des États membres ${ }^{41}$. Un des effets probables de l'harmonisation sera un plafonnement vers le haut des obligations de visa, qui, en même temps, mettra en jeu, dans certains États membres, les liens historiques de dépendance coloniale. C'est le cas en particulier pour la Grande-Bretagne, qui accorde couramment aux nationaux de 48 pays un droit de visite sans visa chez leurs parents ${ }^{42}$. Tel qu'il est expliqué dans le Mémorandum de l'UE concernant la liste de refus proposée, " une liste uniforme... signifie inévitablement que les disparités à ce sujet entre les pratiques des États membres doivent disparaître ". Dans ce but, il a été proposé un régime transitoire unique d'aménagement des " disparités "43. Les contrôles d'inclusion Mise à part la dimension per se de sécurité ${ }^{44}$ exclusionniste, l'aspect inclusionniste présente un différentiel beaucoup plus complexe, en utilisant des séries d'écluses ou de filtres. Les mesures d'inclusion, dans ce contexte, font partie d'une logique économique, qui s'est traduit, par exemple, par des accords d'association et de coopération ${ }^{45}$, conclus entre les pays de la CEE et la Turquie, l'Algérie, le Maroc, le groupe de Visegrád, la Bulgarie... Ces accords impliquaient la venue d'une main d'oeuvre, de préférence bon marché, sans pour autant prévoir ni le droit à la résidence ni d'autres droits civiques ${ }^{46}$. Par ailleurs, des filtres additionnels sélectionnaient ceux qui avaient des qualifications particulières, provoquant ainsi une " fuite des cerveaux ${ }^{47}$ ", ou ceux qui apportaient des capitaux, (le quota de Chinois de Hong-Kong reçus en Grande-Bretagne) ${ }^{48}$. Dans ce contexte, nous pouvons compter parmi ces catégories, d'une part, les " professionnels en transit ", comme les cadres et ceux exerçant des professions libérales expatriés par les compagnies internationales et les programmes $\mathrm{d}^{\prime} \mathrm{aide}^{49}$, et, d'autre part, les travailleurs immigrants illégaux intégrés suite à des amnisties ${ }^{50}$. Cette classification des inclusions peut également être illustrée par une référence au continuum de " droits " civiques accordés par les différents types de statuts de travailleur immigré. Z. Layton-Henry, en 
proposant la typologie suivante, montre que la transition nécessaire à l'obtention des droits civiques passe par l'accueil, le séjour et, finalement, la citoyenneté. STATUTS ET DROITS DES TRAVAILLEURS IMMIGRES ${ }^{51}$ Très peu de droits Peu de droits Sécurité partielle Droits et sécurité appréciables Pleins droits incluant les droits politiques De la même façon, T. Hammar ${ }^{52}$ soutient que les trois principales portes d'entrée, externes et internes, d'un pays d'immigration sont celles qui donnent accès à l'accueil, à la résidence permanente et à la naturalisation. En Allemagne, par exemple, les enjeux spécifiques des droits civiques et la question de la double nationalité ont été soulevés dans la foulée de la violence raciale, à propos des Gastarbeiters ${ }^{53}$ turcs. A. Dumett et J. Niessen ont classifié les inclusions en cinq grandes catégories de " personnes régies par des règles transversales " ${ }^{\circ}$ :

- citoyens de la CEE ;

- citoyens de l'EFTA;

- citoyens des pays ayant conclu des accords d'association ou de coopération (parmi lesquels les droits diffèrent);

- citoyens des pays tiers légalement admis à résider ;

- citoyens des pays tiers ayant obtenu un titre temporaire d'un État membre. La classification des inclusions devient encore plus compliquée si nous prenons en considération les six catégories de nationalité prévues, en Grande-Bretagne, par le Nationality Act de 1981 :

- citoyens britanniques ;

- citoyens britanniques des territoires dépendants ;

- citoyens britanniques d'outre-mer ;

- personnes britanniques protégées ;

- sujets britanniques et, depuis le 1er juillet 1987, nationaux britanniques (outre-mer), à privilège constitué de patrie. Parmi ceux-ci, seuls les citoyens britanniques, les citoyens britanniques des territoires dépendants de Gibraltar et les sujets britanniques ont été considérés comme ressortissants de l'UE ${ }^{54}$. J. Niessen soutient que l'absence de toute volonté politique de réglementation positive de l'immigration dans les pays européens a une influence négative sur le système de protection internationale des réfugiés et sur la contribution virtuelle des immigrants à la solution des problèmes liés au vieillissement des populations ou au rapport entre les classes d'âge actives et inactives ${ }^{55}$. Bien sûr, ceci renforce la marginalisation, tant sociale que juridique, des immigrants et provoque la hausse de l'immigration clandestine. Une main d'oeuvre immigrée non seulement constitue un apport positif à la nation, d'un point de vue économique, mais constitue, en général, un élément vital pour le fonctionnement du système. Cette nécessité politique peut alors être considérée comme une logique économique d'inclusion, ainsi que le soutenait James Callaghan en 1946, bien avant de devenir Premier Ministre : " D'ici quelques années, nous serons confrontés dans ce pays à un manque de demande de travail, et non pas à un manque d'offre d'emplois. Notre taux de natalité n'augmente pas en proportion suffisante pour nous permettre de nous renouveler... Nous rejetons de ce pays des jeunes éligibles et désireux, qui pourraient nous apporter des énergies et des ressources, comme d'autres immigrants ont pu le faire auparavant... Il est peut-être révolutionnaire de suggérer que nous devons devenir un pays accueillant désormais des immigrants, mais ceci serait le développement logique de notre position dans le monde... Qui va payer les retraites et les services sociaux pour les personnes âgées, bénéfices que nous distribuons actuellement, si ce n'est un apport de population que seuls les immigrants pourront assurer dans les jours 
à venir ? "56. L'affirmation de Klaus Kinkel, ministre allemand des Affaires étrangères, sur la " dépendance de l'Allemagne vis-à-vis d'une immigration constante, en raison des conditions économiques et démographiques ", va dans le même sens. Le rapport entre le nombre des retraités et des actifs étant estimé à 1 pour 1 en l'an $2030^{57}$, seul un apport de presque 300000 immigrants pourrait l'équilibrer. Par ailleurs, un organisme gouvernemental belge annonçait, en 1994, que l'entrée annuelle de 100000 immigrants ne pouvait " être que bénéfique pour le système belge de sécurité sociale, vu les prévisions de déséquilibre des retraites et de pénurie de main d'oeuvre "58. Une contradiction logique ? Nous avons suggéré que la notion d'Europe Forteresse, analysée uniquement via l'exclusion, pouvait être davantage examinée car nous constatons en Europe une série de contrôles d'inclusion et d'exclusion, derrière lesquels il y a manifestement deux logiques différentes à l'oeuvre. L'une procède du champ du racisme, de la xénophobie et de la sécurisation, l'autre du champ du protectionnisme économique et de la globalisation du capital. Loin d'être contradictoires, les contrôles d'inclusion et d'exclusion, obéissant à des logiques économiques et sécuritaires, semblent se renforcer réciproquement. Miles affirme par exemple: "Si une Europe Forteresse est à faire, ce ne sont pas seulement les Noirs qui seront rejetés. S'il y a une forteresse qui se construit, elle est conçue pour en interdire l'accès à tous ceux qui cherchent un marché pour leur main d'oeuvre sous-qualifiée ou non-qualifiée, tout comme à ceux qui recherchent un sanctuaire à l'écart des conflits civils et de la répression étatique... il existe une logique de classe, prévalante dans cette structure d'exclusion, logique dont le racisme est une conséquence secondaire, quoique non dépourvue d'importance ${ }^{159}$. Heargraves nous semble résumer parfaitement les principales conséquences du processus étudié, tel que nous l'envisageons, quand il dit: " Les politiciens trouvent peut-être commode de brandir les fanatismes idéologiques ou religieux mais, au fond, les contrôles de l'immigration sont sous-tendus par la terreur qu'inspirent les pauvres du monde... Tant qu'il n'y aura pas une réduction de l'écart entre les conditions matérielles en Europe de l'Est et celles de la CEE... l'ancien rideau de fer restera remplacé par cette nouvelle ligne de séparation entre les nations riches et les nations pauvres en Europe, et celles situées du côté le moins prospère y seront maintenues par les accords négociés à Schengen, à Dublin, à Maastricht... Même si les pays de l'Est atteignent ce seuil, l'UE semble devenir une forteresse obstinément interdite aux pauvres du monde 160 . Nous estimons que c'est cette peur des pauvres qui sous-tend les contrôles d'inclusion et d'exclusion en Europe.

\section{NOTES}

1. Mike King est Lecturer au Scarman Centre de l'Université de Leicester.

2. Cet article est une version mise à jour et révisée d'un papier présenté à l'Université de Leicester. L'auteur tient à remercier tous ceux qui ont contribué à sa rédaction.

3. D. Thränhardt et R. Miles, Migration and European Integration : the dynamics of inclusion and exclusion, London, Pinter, 1995, introduction, pp. 1-12. 
4. Ces catégories ne se recoupent pas entièrement. Bien que l'article 14 de la Déclaration Universelle des Droits de l'Homme proclame que " tout être humain a le droit de demander asile dans un autre pays s'il est persécuté ", la notion de persécution justifiant la reconnaissance du statut de réfugié à une personne de bonne foi, varie suivant les accords internationaux et régionaux. La définition du " réfugié " de l'article 1 de la Convention de Genève sur le statut des réfugiés de 1951, amendée par le Protocole de New York de 1967, est plus étroite que celle contenue dans l'article 1(2) de la Convention sur les problèmes des réfugiés en Afrique de l'OUA de 1969. Sont inclus de facto dans les réfugiés, tous ceux qui sont contraints à des migrations forcées du fait des guerres et des famines. J. Doomernik, R. Penninx et H. van Amersfoort suggèrent qu'ils appartiennent à une autre catégorie de migrants inextricablement liée par un processus économique (et pas simplement par les guerres, les famines, les persécutions raciales) aux contrôles exclusionnistes. Voir M. King, " The impact of Western European Border Policies on the Control of Refugees in Eastern and Central Europe ", New Community, 1993, vol. 19, n² 2, p. 191 ; " Policing Refugees and Asylum seekers in "Greater Europe" : towards a reconceptualisation of control " in Anderson \& den Boer, Policing Across National Boundaries, Londres, Pinter, 1994, p. 73 ; M. Spencer, States of Injustice $^{\circ}$ : a guide to Human Rights and civil liberties in the European Union, London, Pluto Press, 1995, pp. 76-104 ; G. Goodwin-Gill, The Refugee in International Law, Oxford, 1996, pp. 3-31 et J. Doomernik, R. Penninx and H. van Amersfoot, A migration policy for the Future : possibilities and limitations, Brussels, Migration Policy Group, 1997.

5. M. King, " The impact... ", op. cit., pp. 183-189 et " Policing Refugees... ", op. cit., pp. 69-84.

6. Ce différentiel d'inclusion est lié intrinsèquement aux contrôles d'exclusion de l'immigration, quelles que soient leurs formes : contrôles frontaliers, internes (lieux de résidence, lieux de travail) ou, comme nous le verrons plus loin, par une série de filtres. Voir J. Niessen and R. Z. de Beij, " International Migration Economic development and Human Rights ", Churches Commission for Migrants in Europe (CCME), Briefing Paper $\mathrm{n}^{\circ} 18$, Brussels, 1994, p. 7.

7. Frankfurter Allgemeine Zeitung, 22.05.1993, traduction de l'auteur.

8. Le groupe de Visegrád comprend la République Tchèque, la Hongrie, la Pologne et la Slovaquie.

9. Statewatch, nov.-déc.1993, p. 17.

10. Idem, sept.-oct. 1993.

11. En octobre 1993, par exemple, deux Kurdes furent renvoyés en Grande-Bretagne par les autorités néerlandaises, après avoir été expulsés de Grande-Bretagne vers les PaysBas, selon la règle du pays tiers. En septembre 1993, le Bundesverfassungsgericht a estimé, par deux décisions en référé, que trois demandeurs d'asile iraniens pouvaient déposer leur demande en Allemagne plutôt que d'être renvoyés en Grèce suivant la règle du premier pays d'asile. Dans un autre cas, une instance judiciaire britannique a estimé que la Suède ne pouvait pas être considérée comme un pays sûr pour les Albanais du Kosovo ; malgré tout, le demandeur d'asile en question a été renvoyé en Suède par la suite. Voir sur ces points E. Pilkington, " Dutch return Kurds deported under "Third Country" Rule ", The Guardian, 1er nov. 1993, et MNS, oct. 1993 et fév. 1994.

12. A. Cruz signale ici une contradiction de terminologie. La version anglaise de l'accord de Schengen et de la Convention sur le contrôle des personnes aux frontières 
extérieures fait état de pénalités plutôt que de sanctions. Aucun des deux textes ne fait mention d'une obligation d'amende. A. Cruz, " Carrier Liability in the Member States of the European Union ", Churches Commission for Migrants in Europe, Briefing Paper $\mathrm{n}^{\circ}$ 17, Brussels, CCME.

13. De telles mesures ne sont pas réellement nouvelles : elles ont été appliquées aux compagnies de navigation du fait de l'Aliens Act de 1905, (sauf aux passagers de première classe !). Voir R. Miles, " Who belongs ? : the meaning of the British Nationality and Immigration Law ", Journal of Law and Society, vol. 18, $\mathrm{n}^{\circ} 2$, pp. 279-286. L'amende initiale ( $1000 £$ par passager clandestin) est passée à $2000 £$ en 1991. En 1993, le montant de ces amendes a totalisé 55 millions de livres. Voir l'article de N. Davies, " The Death Ship ", The Guardian Weekend, 23.10.1993, pp.16-23.

14. A. Cruz les juge parfaitement incompatibles avec les articles 31,31(2) et 33 de la Convention de Genève. A. Cruz, op. cit., pp. 16-17.

15. Un accord de réadmission a été signé entre l'Allemagne et la Roumanie le 24 septembre 1992, prévoyant le retour par une " procédure simplifiée " des ressortissants roumains ; un autre l'a été avec la Bulgarie le 12 novembre 1992, incluant le versement par l'Allemagne de 28 millions de marks. Voir MNS, avril 1993 ; M. Schieffer, "The Readmission of Third Country Nationals within Bilateral and Multilateral Frameworks ", communication au quatrième colloque de l'Institut Européen d'Administration publique, Schengen and the Third Pillar of Maastricht, Maastricht, 1er et 2 fév. 1996. 16. Ministère de l'Intérieur, Circulaire $n^{\circ} 8$, à Messieurs les Préfets de la Métropole : Accord de refoulement franco-allemand, 30 janvier 1960 ; ces accords concernant " la prise en charge des personnes à la frontière " ont été renouvelés en 1962, 1964 et 1965, cf. ministère de l'Intérieur, Circulaire $\mathrm{N}^{\circ}$ NOR/INT/D/93/00210/C : Accords bilatérauxpays européens, 8 septembre 1993.

17. M. Schieffer, op. cit., p. 4.

18. Idem, pp. 4-5 ; M. Spencer, op. cit., p. 62.

19. La Suisse a ainsi signé avec la Hongrie un accord bilatéral le 05.02.1994. Il semble que l'intérêt de cet accord pour les Hongrois est de devenir à leur tour un pays de transit, ce qui leur permet de... rapatrier les Albanais du Kosovo, que les Suisses n'acceptent plus, et " que la Macédoine refuse de laisser transiter sur son territoire ", MNS, mars 1994, p. 8.

20. Statewatch, nov-déc. 1993, p. 14 ; MNS, mars 1994, nº 2.

21. Spencer, op. cit., p. 90.

22. Schieffer, op. cit., p. 6.

23. Le nombre de demandeurs d'asile, refoulés fin janvier 1994, était évalué entre 2400 et 6400 ; le second chiffre inclut, tous ceux qui ont été arrêtés en train de franchir illégalement la frontière germano-polonaise. Un accord similaire fonctionnant sur la base d'un quota annuel a été mis en place pour rapatrier les 40000 Vietnamiens exGastarbeiters de l'ex-R.D.A., pris au piège après la réunification ; MNS, fév. 1994, p. 6 ; Schieffer, op. cit., p. 8.

24. Parlement Européen, Second report of the Committee on Civil Liberties and International Affairs on the entry into force of the Schengen Agreements (Rapporteur L. van Outrive), 5 novembre 1992, p. 29 ; S. Castles and M. Miller, The Age of Migration : international migration movements in the modern world, London, Macmillan, 1993, p. 127 ; M. King, " Policing Refugees... ", op. cit., p. 1.

25. MNS, sept. 1993, p. 3.

26. Idem, p. 11. 
27. C. Wallace, O. Chmouliar et E. Sidorenko font référence à une " frontière invisible ", franchie journellement par plus d'un demi-million de personnes, dans le sens EstOuest : C. Wallace, O. Chmouliar and E. Sidorenko, " The Eastern Frontier of Western Europe : mobility in the buffer-zone ", New Community, 1996, vol. 22, n² 2, pp. 259-286. 28. M. Schieffer, op.cit., p. 10.

29. A. Funk cite les commentaires de la Radio Prague sur l'amendement A16a à la Constitution allemande : "ol'un après l'autre, chaque pays fermera ses frontières du fait des réfugiés des pays voisins. Plus ils sont loin à l'Est, plus les "dominos" tomberont... " in A. Funk, " Control Myths : the Eastern border of the Federal Republic of Germany before and after 1989 ", intervention à l'ECPR, workshop " Police and Immigration Towards a Europe of Internal Security ", Madrid, 18-22 avril 1994, p. 15.

30. La Hongrie a conclu des accords de réadmission avec tous ses voisins, à l'exception de l'ex-Yougoslavie, cf. B. Nagy, " Changing Trends, Enduring Questions regarding Refugee Law in Central Europe " in M. Fullerton, E. Sík and J. Tóth (eds), Refugees and Migrants : Hungary at Crossroads, Budapest, 1995, IPSHAS, pp. 27-54.

31. R. McDowall observe que le seul effet du régime des visas et des sanctions contre les convoyeurs est une déviation, cf. R. McDowall, " Coordination of refugee Policy in Europe " in G. Loescher and L. Monahan (eds), Refugees and International relations, Oxford, Clarendon Press, 1990, pp. 179-186.

32. MNS, fév. 1994, p. 6.

33. Bundeszentrale für politische Bildung, Informationen zur politischen Bildung : Ausländer, 1992, n 237, 4. Quartal, p. 39.

34. Statewatch, sept-oct. 1993, p. 2.

35. J. Carvel, " Changing Face of EC Refugees ", The Guardian, 01.06.1993.

36. MNS, août 1996.

37. MNS, fév. 1994, p. 6 ; Statewatch, nov.-déc. 1993, p. 15.

38. MNS, sept. 1993, p. 4. En 1993, 35399 demandes d'asile furent déposées aux PaysBas, contre 20346 en 1992, soit une augmentation de 74\%. Voir MNS, fév. 1994, p. 12 et J. Doomernik, R. Penninx and H. van Amersfoot, op. cit., p. 35.

39. De ce point de vue, M. den Boer estime qu'il y aura une "imperméabilité croissante de l'Europe ", qui se reflète déjà dans la métaphore de l'Europe Forteresse et dans le respect des accords signés par les gouvernements de l'UE. Un fait qui décentre la " stratégie intégrationniste de la Commission européenne. Un exemple classique de ce fait est l'opinion exprimée par l'ancien Premier Ministre M. Thatcher : " Nous avons rejoint l'Europe pour bénéficier de la liberté de mouvement des marchandises... nous n'avons pas rejoint l'Europe pour avoir la liberté de circulation des terroristes, des criminels, des stupéfiants, des maladies animales et végétales et des immigrants illégaux ", cité par M. Baimbridge, B. Burkitt and M. Macey, " The Maastricht Treaty : exacerbating racism in Europe ? ", article non publié. Voir aussi M. den Boer, " Moving between the Bogus and Bona Fide ${ }^{\circ}$ : the policing of inclusion and exclusion in Europe " in R. Miles and D. Thränhardt, op. cit., pp. 92-111.

40. Annexe du communiqué CEE, 1993.

41. CEE, 1993, mémorandum d'explication.

42. J. Carvel, " Queen Urged to Fight EC Visa Plan ", The Guardian, 03.11.1993.

43. Communiqué CEE 1993, Proposition pour une régulation sous A. 100C, p. 1.

44. Conceptualisée par D. Bigo en termes de " continuum de sécurité intérieure ", D. Bigo, " The European Internal Security Field : stakes and rivalries in a newly developing 
area of police intervention " in M. Anderson and M. den Boer, Policing Across National Boundaries, London, Pinter, 1994, pp. 161-173.

45. Il existe trois catégories d'accords : un accord d'association avec la Turquie; des accords de coopération avec l'Algérie, la Tunisie, le Maroc et l'ancienne Yougoslavie (ce dernier suspendu) ; enfin, des accords " mixtes " avec les pays du groupe de Visegrád, la Roumanie et la Bulgarie. Ces accords datent de 1963 et 1978 pour l'Algérie et le Maroc; de 1991 et, puis, d'octobre 1993 pour la nouvelle République Tchèque et de mars de la même année pour la Slovaquie. Voir E. Guild, " Protecting Migrants'Rights : application of EC agreements with third countries ", Churches Commission for Migrants in Europe (CCME), Briefing paper n 10, Brussels, 1992, pp. 3-20 ; MNS, avril 1993, p. 11 ; A. Dummet and J. Niessen, " Immigration and Citizenship in the European Union ", Churches Commission for Migrants in Europe and the Commission for Racial Equality, Briefing Paper $n^{\circ} 14$, Brussels, 1993.

46. En général, les accords stipulent formellement que " au regard des conditions de travail et de rémunération, les règles que chaque État membre applique aux travailleurs concernés... employés dans la Communauté ne doivent pas être discriminatoires sur le plan de la nationalité entre ces travailleurs et ceux des États membres de la Communauté ", E. Guild, op. cit., p. 3. Les accords avec le groupe de Visegrád et les pays d'Europe centrale et de l'Est retiennent cette provision, mais à la seule condition que " ces travailleurs soient employés légalement sur le territoire d'un État membre ", E. Guild, op. cit., p. 18.

47. V. Zlatanova, " The Bulgarian Brain Drain ", Migration, Berlin, 1991, n 11-12, pp. 133-142.

48. Un bon exemple de ce différentiel est fourni par les Chinois de Hong-Kong (50 000 par an) qui furent autorisés jusqu'en mars 1996 à demander la citoyenneté britannique (territoires dépendants) sous le bénéfice du British Nationality (Hong-Kong) Act de 1990. R. Miles, op. cit., p. 284.

49. S. Castles et M. Miller, op. cit., p. 161.

50. Z. Layton-Henry, " Citizenship or Denizenship for Migrant Workers ", p. 190, in Layton-Henry (ed)., The Political Rights of Migrant Workers in Europe, London, Sage, 1990.

51. Source : Z. Layton-Henry, op. cit., p.14.

52. T. Hammar, "The Democratic Nation-State and the Challenge of Migration ", Papers presented to the Sam.Pol. Konferansen 1993, Migration : The politics of contemporary population movements, Bergen, 1993, pp. 72-80.

53. D. Gow, " Kohl Threatens Turk Extremists ", The Guardian, 02.06.1993 ; T. Sommer, " Darkness that Breaks the Chain of light ", The Guardian, 04.06.1993 ; A. Dummett and J. Niessen, op. cit., p. 25.

54. J. Niessen and R. Z. de Beij, " International Migration, Economic development and Human Rights ", CCME, Briefing Paper n' 18, p. 5.

55. J. Niessen, " The Making of European Immigration Policies ", CCME, Briefing Paper $\mathrm{n}^{\circ} 15$, p. 5.

56. R. Cohen, The New Helots : migrants in the international division of labour, Avesbury, 1987, p. 124.

57. MNS, sept. 1993, p. 2.

58. MNS, mars 1994, p. 10.

59. R. Miles, Racism after "Race relations", London, Routledge, 1993, p. 18. 
60. A. Heargraves, " Migration Controls, Open Frontiers and European unity ", Journal of Area Studies,1992,n¹,pp.84-85.

INDEX

Mots-clés : contrôle, économie politique, racisme 